\title{
Charm Fragmentation Function and Charm Fragmentation Fractions at ZEUS
}

\author{
Shuangshi Fang \\ (ON BEHALF OF THE ZEUS COLLABORATION) \\ DESY,Notkestasse 85, Hamburg 22607, Germany
}

\begin{abstract}
Based on the data collected during 1996-2000 period at ZEUS detector, the charm fragmentation function and fragmentation fractions have been measured in photoproduction and deep inelastic scattering,respectively. The measured function is compared to different fragmentation models incorporated in leading-logarithm Monte Carlo simulations and a next-to-leading-order calculation. The shape is similar to those from $e^{+} e^{-}$experiments. The measured charm fractions are consistent with previous measurements.
\end{abstract}

\section{Introduction}

Heavy quark production offers a sensitive test of QCD predictions. In this talk[1] "heavy quark" refers to a charm(anti-charm) quark.Usually heavy quark production was divided into four steps: the production of heavy quark pairs; the development of parton showers; the transition of partons to hadrons(also known as fragmentation) and the unstable hadrons decay according to their branching fractions. The first two steps can be calculated with $\mathrm{pQCD}$, while the fragmentation is a non-perturbative process. The phenomenological models[2] have been applied to study charm fragmentation and two widely used fragmentation functions are the model of Peterson et al.[3] and of Kartvelishvili et al.[4]. Since these non-perturbative models are not calculated from the first principle, experimentally study is necessary to determine the parameters of the fragmentation functions.

Experimentally, charm fragmentation functions have been studied for many years[5] in $e^{+} e^{-}$annihilation. It also has been studied in ep collisions by H1 collaboration[6]. The most recent published results with high precision is from Belle[7]. For a recent review of fragmentation function measurements and theory, see[8].

Meanwhile, the measured production cross sections of $D^{0}, D^{+}, D^{*+}$ and $D_{s}^{+}$charm mesons allow to extract charm fragmentation fractions which describe the probability of charm quark hadronsing into particular charm mesons. In this talk, we present the recent preliminary results for fragmentation functions and the measurement of fragmentation fractions based on HERAI data taken at ZEUS detector. The ZEUS detector is a multipurpose magnetic detector designed to study $e p$ scattering at HERA which is described in detail in Ref.[9].

\section{Charm fragmentation function}

The data collected during the 1996 to 2000 running period corresponding to an integrated luminosity of $120 \mathrm{pb}^{-} 1$ was used for this analysis.

The measurement was performed in the kinematic range $Q^{2}<1 \mathrm{GeV}^{2}$ and $130<$ $W_{\gamma p}<280 \mathrm{GeV}$. The jets were reconstructed using the $\kappa_{T}$ algorithm with at least one jet required to have $E_{T}^{\text {jet }}>9 \mathrm{GeV}$ and $\left|\eta^{\text {jet }}\right|<2$.4. The $D^{*}$ mesons were reconstructed using 
$D^{*} \rightarrow D^{0} \pi_{s} \rightarrow K \pi \pi_{s}$. The transverse momentum of $D^{*}$ was required to be greater than $2 \mathrm{GeV}$ and the pseudo-rapidity was required to be in the region $\left|\eta^{D^{*}}\right|<1.5$. To minimise background, narrow windows were selected for the mass difference and the mass the $D^{0}$ meson: $0.1435<\Delta M<0.1475 \mathrm{GeV}$ and $1.83<M\left(D^{0}\right)<1.90 \mathrm{GeV}$. Finally the $D^{*}$ meson as associated with a jet by considering the closest jet in $\eta-\phi$ space and requiring $\mathrm{R}\left(=\sqrt{\left(\eta^{j e t}-\eta^{D^{*}}\right)^{2}+\left(\phi^{j e t}-\phi^{D^{*}}\right)^{2}}\right)<0.6$. After above selection and subtraction of the background bin-by-bin estimated from wrong-charge pairs, $1268 \pm 56 \mathrm{D}^{*}$ mesons were used for further analysis.

Since the energy of charm quark is approximated by the energy of the reconstructed $D^{*}$ jet, the fragmentation observable $\mathrm{z}$, the energy transferred from a charm quark to a given meson, was defined as $\left(E+p_{\|}\right) / 2 E_{j e t}$, where the $p_{\|}$is the longitudinal momentum of the $D^{*}$ relative to the jet axis. Two Monte Carlo(MC) simulations(PYTHIA 6.1 and HERWIG 6.1) were compared with the $\mathrm{z}$ of the data in which PYTHIA seems to be better in describing data. The data was then corrected for detector effects using a bin-by-bin method with the PYTHIA simulation used as the central MC and the HERWIG simulation as a systematic check.

The relative cross sections were also used to extract the fragmentation parameter of Peterson function. The MC was fit to the data via a $\chi^{2}$-minimisation procedure to determine the best value of $\varepsilon$. The result of varying $\varepsilon$ is shown in Fig. 1 and the data is well described by the best value, $\varepsilon=0.064 \pm 0.006_{-0.008}^{+0.011}$, of the fit(for the figures of the following fit, see[1]).

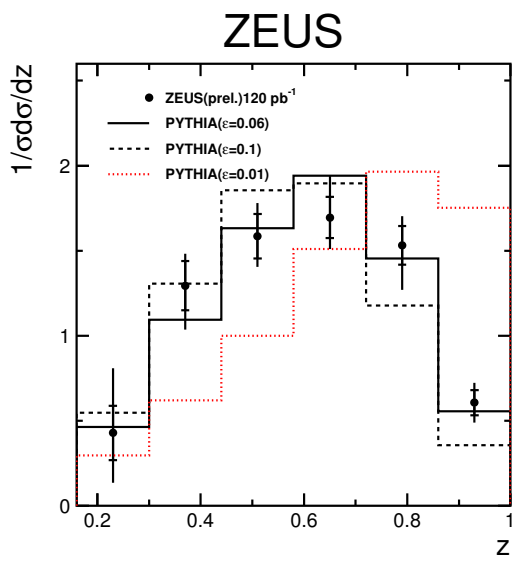

Figure 1: Relative cross section, $1 / \sigma(d \sigma / d z)$, for the data(points) compared with values of parameter $\varepsilon=1$ (dashed line), $\varepsilon=0.06$ (solid line) and the $\varepsilon=0.02$ (dotted line), in the Peterson fragmentation function as as implemented in PYTHIA predictions.

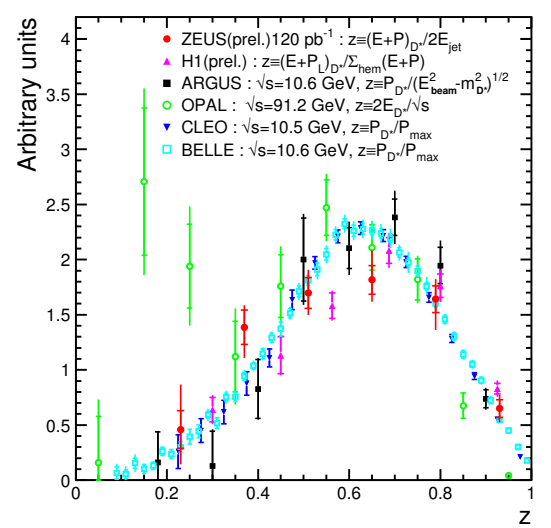

Figure 2: Fragementation function versus z for the ZEUS data compared to previous measurements. For shape comparison, the data sets were normalized to $1 /$ (bin width) for $z>$ 0.3 , thus avoiding the first three bins from OPAL, which have large gluon-splitting component.

Similarly, the parameters of Peterson function and Kartvelishvili function were extracted in the next-to-leading order(NLO) framework[11], respectively. As the final state particles 
in the NLO QCD calculation are partons, to enable a fair comparison with the data, the predictions were corrected for effects of hadronization. The fit to the data gives the best value of $\varepsilon=0.0721_{-0.0123}^{+0.0139}$ and $\alpha=2.87_{-0.35}^{+0.33}$.

The comparison of the ZEUS data with previous measurements is shown in Fig. 2. To avoid the first three bins from OPAL collaboration which have a large gluon-splitting component, the data sets were normalised to $1 /$ (bin width) for $z>0.3$. Although the definitions of fragmentation observable and kinematics range are different, the spectra are similar in shape.

\section{Charm fragmentation fractions}

Charm production in deep inelastic scattering has been measured with the ZEUS detector at HERA using an integrated luminosity of $82 \mathrm{pb}^{-1}$. Charm has been tagged by reconstructing $D^{*}+, D^{0}, D^{+}$and $D_{s}^{+}(+$c.c. $)$charm mesons. The charm hadrons were measured in the kinematic range $p_{T}\left(D^{*+}, D^{0}, D^{+}, D_{s}^{+}\right)>3 \mathrm{GeV}$ for $1.5<Q^{2}<1000 \mathrm{GeV}^{2}$ and $0.02<y<0.7$. The production cross sections were used to extract charm fragmentation ratios and the fraction of charm quarks haronising into a particular charm meson in the kinematic range considered. The detailed description of event selection are listed in Ref.[12].

The fragmentation fractions of charm quark hadronising to a specific charm meson are listed in Table. 1. The results are compared with the values obtained in photoproduction[13], in DIS by the H1 collaboration[14] and in $e^{+} e^{-}$annihilations[15]. All the measurements are consistent with each other.

\begin{tabular}{|c|c|c|c|}
\hline$f\left(c \rightarrow D^{+}\right)$ & $0.216 \pm 0.019_{-0.020-0.010}^{+0.002+0.008}$ & $R_{u / d}$ & $1.22 \pm 0.11_{-0.002}^{+0.005} \pm 0.03$ \\
\hline$f\left(c \rightarrow D^{0}\right)$ & $0.605 \pm 0.020_{-0.052-0.023}^{+0.009+0.015}$ & $\gamma_{s}$ & $0.225 \pm 0.030_{-0.007-0.026}^{+0.018+0.034}$ \\
\hline$f\left(c \rightarrow D_{s}^{+}\right)$ & $0.092 \pm 0.011_{-0.008}^{+0.007+0.012}$ & $P_{V}^{d}$ & $0.617 \pm 0.038_{-0.009}^{+0.017} \pm 0.017$ \\
\hline$f\left(c \rightarrow D^{*+}\right)$ & $0.229 \pm 0.011_{-0.021-0.010}^{+0.006-0.007}$ & & \\
\hline
\end{tabular}

Table 1: The fractions of charm quarks hadronising as a particular charm meson and the results for $R_{u / d}, \gamma_{s}$ and $P_{V}^{d}$.

In addition to charm fragmentation fractions, the fragmentation ratios shown in Table. 1 are calculated based on the measured production cross sections of charm mesons. The ratio of neutral to charged $\mathrm{D}$ meson production, $R_{u / d}$, is used to test the iso-spin invariance. It is consistent with 1 , confirming the iso-spin invariance, which implies that $\mathrm{u}$ and $\mathrm{d}$ quarks are produced equally in charm fragmentation. The strangeness-suppression factor for charm mesons, $\gamma_{s}$, is given by the ratio of twice the production rate of charm-strange mesons to the production rate of non-strange charm meson. And the strange quark production is measured to be suppressed by a factor $\sim 4$ as shown by $\gamma_{s}$. The ratio of charged D mesons produced in a vector state, $P_{V}^{d}$, is given by the ratio of vector to the sum of vector and pseudo-scalar charm meson production cross sections. The measured $P_{V}^{d}$ value is smaller than the naive spin counting prediction of 0.75 . The ratios are in good agreement with previous measurements[13][14][15][16]. 


\section{Summary}

Charm fragmentation function has been studied in photoproduction regime with HERAI data. The parameters of Peterson and Kartvelishvili functions were extracted. The study indicates that both of the fragmentation functions provide a reasonable description of data. Comparison with previous measurements shows that the spectra are similar in shape although the fragmentation observable definitions and kinematics are different.

Charm fragmentation fractions were measured in DIS regime and the results are in good agreement with those measured in photoproduction regime and in $e^{+} e^{-}$annihilation. $R_{u / d}$, $\gamma_{s}$ and $P_{d}^{V}$ were measured which are also consistent with previou smeasurements. $R_{u / d}$ is compatible with unity which indicates the iso-spin invariance, but $P_{V}^{d}$ was found to be smaller than the naive spin-counting prediction of 0.75 .

\section{References}

[1] http://indico.cern. ch/contributionDisplay $\cdot$ py? contribId=193\&sessionId=5\&conf Id=9499

[2] P.D.B. Collins and T.P. Spiller, J. Phys. G11,1289(1985);

B. Andersson, G. Gustafson, and B. Söderberg, Z. Phys. C20,317(1983);

M.G. Bowler, Z. Phys. C11,169(1981).

[3] C. Peterson, D. Schlatter, I. Schmitt, and P.M. Zerwas, Phys. Rev. D27,105(1983).

[4] V.G. Kartvelishvili, A.K. Likhoded, and V.A. Petrov, Phys. Lett. 78B,615(1978).

[5] ARGUS Coll., H. Albrecht et al., Z. Phys. C52,353(1991); OPAL Coll., R. Akers et al., Z. Phys. C67,27(1995); CLEO Coll., M. Artuso et al., Phys. Rev. D70,112001(2004).

[6] H1 Coll., Determination of the Charm Fragmentation function in Deep-Inelastic Scattering at HERA, contributed paper to XXIInd International Symposium on Lepton-Photon Interactions at High Energy, June 2005, Uppsala, Sweeden. (unpublished). 2005.

[7] Belle Coll., R. Seuster et al., Phys. Rev. D73,032002(2006).

[8] O. Biebel, P. Nason, and B. R. Webber, hep-ph/0109282.

[9] ZEUS Coll. M. Derrick et al., Phys. Lett. B297,404(1992).

[10] S. Nussinov, Phys. Rev. Lett. 35,1672(1975);

G. J. Feldman et al., Phys. Rev. Lett. 38,1313(1977).

[11] S. Frixione et al., Phys. Lett. B348,633(1995);

S. Frixione, P. Nason and G. Ridolfi, Nucl. Phys. B454,3(1995).

[12] ZEUS Coll., S. Chekanov et al., e-Print: arXiv:0705.1931 [hep-ex].

[13] ZEUS Coll., S. Chekanov et al., Eur. Phys. J. C44,351(2005).

[14] H1 Coll., A. AKtas et al., Eur. Phys. J. C38,447(2005).

[15] L. Gladilin, Preprint hep-ex/99120674,1999.

[16] ZEUS. Coll., J. Breitweg et al., Phys. Lett. B481,213(2000). 